\title{
PTSD: past, present, and future
}

\author{
Bret A. Moore* \\ Warrior Resiliency Program, United States Army Southern Regional Medical Command, San Antonio, Texas, USA
}

Received 13 February 2013; Accepted 15 February 2013

Key words: posttraumatic stress, evidence based treatment, psychotherapy research, psychopharmacology research, integrated treatment.

The addition of posttraumatic stress disorder (PTSD) to the third edition of the Diagnostic and Statistical Manual of Mental Disorders (DSM-III) ${ }^{1}$ was a significant advancement in the fields of psychiatry and psychology. Securing a formal slot in the American Psychiatric Association's official nosological classification scheme increased the diagnostic validity and fidelity of the disorder, and also paved the way for advancements in clinical and translational research. This milestone, however, does not negate the data that have been gleaned from military conflicts spanning the time from the ancient Battle of Marathon to the end of the Vietnam War. In some ways, functioning outside of a classification system has allowed clinical scholars to explore the complexity of humans' reactions to trauma, better understand the individual differences associated with psychological resilience and recovery, and develop common sense intervention strategies spawned by necessity and compassion. Our past with PTSD is both long and important.

Today, our patients reap the benefits from what we have learned about PTSD through research and practice. Advances in understanding the causes and nature of PTSD have allowed us to refine our definition of the disorder. In addition to removing the diagnosis from the broad category of Anxiety Disorders in the DSM and creating a more appropriate placement, labeled Trauma- and Stressor-Related Disorders, we are questioning the validity of various diagnostic criteria. For example, it has been proposed that diagnostic criterion A2 does not always apply to combat-related trauma, as training and other occupational factors unique to military service provide a substantial buffer against fear, helplessness, or horror. ${ }^{2}$ The outcome data from psychotherapy studies are hitting scientific journals and professional conferences

\footnotetext{
*Address for correspondence: Bret A. Moore, Warrior Resiliency Program, 7800 IH 10 West, Suite 300, San Antonio, TX 78230, USA. (Email: bret.moore@us.army.mil)

The views presented in this editorial are those of the author and do not reflect the official policy or position of the U.S. Army, the Department of Defense, or the U.S. government.
}

at a breakneck pace. More importantly, the data are positive. "Top-tier" psychotherapy treatments such as prolonged exposure and cognitive processing therapy are showing remarkable results $^{3-5}$; however, other psychotherapies tend to maintain their robust effects within meta-analyses. ${ }^{6}$ There are risks to scientific and clinical progression. As PTSD psychotherapy researchers and practitioners continue to identify with different psychotherapy "camps" and financial motives-whether through grant funding or proprietary interests-potentially stymie objective collection, comparison, and interpretation of outcome data, we run the risk of overestimating the effects of some treatments while minimizing the effects of others. Ensuring that bona fide comparison groups are relied upon in head-to-head trials is one way to mitigate this risk. Pharmacotherapy has also proven itself to be an important component of effective PTSD treatment. In a systematic review of 35 short-term randomized controlled trials (RCTs) including over 4500 PTSD patients, medication provided significant benefits compared to placebo. ${ }^{7}$ Moreover, benefits extended beyond traditional PTSD symptoms, and gains were seen with depression and level of disability. Selective serotonin reuptake inhibitors (SSRIs) have become the first-line pharmacological treatment of PTSD for good reason. Meta-analyses and several RCTs support the superiority of SSRIs over placebo. Studies with serotonin-norepinephrine reuptake inhibitors (SNRIs) have resulted in similar positive effects. ${ }^{8}$ One important caveat is that positive treatment effects, particularly those reaching the level of remission or converting a nonresponder to a responder, are directly related to duration of treatment. In the case of pharmacotherapy, longer tends to be better. There is a flipside, however. For every positive medication study, there seems be a neutral or negative one ready to counter. Also, outside of SSRIs and SNRIs, the research supporting other medication classes (atypicals and anticonvulsants) is scant, even disappointing in some cases. This is concerning, considering how common off-label medications, many with potential serious side effects, 
are used without an acceptable level of scientific support based on rigorous controlled studies. Case reports, open label trials, and clinical anecdotes only go so far.

The future is bright ... potentially. Psychiatry and psychology are in ideal positions to develop, expand, and refine biological and psychosocial treatments for PTSD. However, to do so requires adequate funding, earnest scientific inquiry, and lessening the divide between camps, specialties, and societies. The field of psychotherapy research would benefit from placing emphasis on dismantling studies that look at the core components of effective treatments as opposed to endeavoring to "prove" why one treatment is better than the other, especially when we have data showing they are all equally effective when bona fide comparisons are employed. ${ }^{6}$ Instead of attempting to parse out unique aspects of a treatment for the purpose of separating from the pack, focus should be on identifying common factors that are surely inherent in most if not all effective psychotherapies. Another likely fruitful area of pursuit in psychotherapy research is further understanding how psychotherapy affects neurobiology. It is quite possible that medication is not the only intervention that improves efficiency of information processing in hypothetically malfunctioning brain circuits. ${ }^{9}$ Psychopharmacology research has room for growth as well. Investment, both financially and intellectually, in identifying novel pharmacological interventions is crucial to advancement. Instead of focusing on whether or not fluoxetine is better than escitalopram for relieving global symptoms of PTSD, or developing "me too" drugs that offer little if any additional benefit beyond that of their predecessors, exploring the role of glucocorticoids, NMDA antagonists, and cannabinoids may be more fruitful. Then there is the issue of combined treatments. The literature on the effectiveness of integrating pharmacological and psychological treatments is scarce. This is likely the most untapped area of investigation with the most potential payoff. ${ }^{10}$

The current issue of CNS Spectrums has increased the candlepower with regard to illuminating the future of effective pharmacological and psychological treatments for PTSD, as well as our understanding of this complex disorder. In this issue, researchers and clinical scholars address important issues such as attentional differences between PTSD patients and healthy controls, biopsychosocial implications of manmade versus natural disasters, effects of hydrocortisone on the prevention of PTSD, and enhancing current evidencebased psychological interventions. Dr. Stahl and the editorial board should be commended for such forward thinking. Hopefully, in part, this issue will fuel continued scientific and clinical curiosity, and investigation into remedies for a disorder that has taken national and international center stage within the discourse of psychiatry and psychology.

\section{References}

1. American Psychiatric Association. Diagnostic and Statistical Manual of Mental Disorders, 3rd ed. Washington, DC: American Psychiatric Association; 1980.

2. Adler A, Wright K, Bliese P, Eckford R, Hoge C. A2 diagnostic criterion for combat-related posttraumatic stress disorder. J Trauma Stress. 2008; 21(3): 301-308.

3. Powers M, Halpern J, Ferenschak M, Gillihan S, Foa E. A meta-analytic review of prolonged exposure for posttraumatic stress disorder. Clin Psychol Rev. 2010; 30(6): 635-641.

4. Williams A, Galovski T, Kattar K, Resick P. Cognitive processing therapy. In: Moore BA, Penk WE, eds. Treating PTSD in Military Personnel: A Clinical Handbook. New York: Guilford Press; 2011: 59-73.

5. Chard KM. An evaluation of cognitive processing therapy for the treatment of posttraumatic stress disorder related to childhood sexual abuse. J Consult Clin Psychol. 2005; 73: 965-971.

6. Benish S, Imel Z, Wampold B. The relative efficacy of bona fide psychotherapies for treating post-traumatic stress disorder: a meta-analysis of direct comparisons. Clin Psychol Rev. 2008; 28(5): 746-758.

7. Stein DJ, Ipser JC, Seedat S. Pharmaotherapy for posttraumatic stress disorder (PTSD). Cochran Database Syst Rev. 2006; 1: CD002795.

8. Benedek DM, Friedman MJ, Zatzick D, Ursano RJ. Practice guideline for the treatment of patients with acute stress disorder and posttraumatic stress disorder. Psychiatry Online. 2009. DOI: 10.1176/appi.books. 9780890423479.156498. Retrieved from http:/ / www.psychiatryonline.com/content.aspx?aid $=156498$

9. Stahl SM. Psychotherapy as an epigenetic "drug": psychiatric therapeutics target symptoms linked to malfunctioning brain circuits with psychotherapy as well as with drugs. J Clin Pharm Ther. 2012; 37: 249-253.

10. Stahl SM, Moore BA. Anxiety Disorders: A Guide for Integrating Psychopharmacology and Psychotherapy. New York: Routledge Press; 2013. 